\title{
LIMITS OF PERFORMANCE IN REFERENCE-TRACKING AND PATH-FOLLOWING FOR NONLINEAR SYSTEMS
}

\author{
A. Pedro Aguiar ${ }^{*, 1}$ João P. Hespanha ${ }^{*, 2}$ \\ Petar Kokotović ${ }^{*}, 1$ \\ * Center for Control Engineering and Computation \\ University of California, Santa Barbara, CA 93106-9560 \\ \{aguiar, hespanha, petar\}@ece.ucsb.edu
}

\begin{abstract}
We investigate limits of performance in reference-tracking and pathfollowing and highlight an essential difference between them. For a class of nonlinear systems, we show that in reference-tracking, the smallest achievable $\mathcal{L}_{2}$ norm of the tracking error is equal to the least amount of control energy needed to stabilize the zero-dynamics of the error system. We then show that this fundamental performance limitation does not exist when the control objective is to force the output to follow a geometric path without a timing law assigned to it. This is true even when an additional desired speed assignment is required to be satisfied asymptotically or in finite time.
\end{abstract}

Keywords: Limits of performance, non-minimum phase nonlinear systems, path-following, reference-tracking, cheap-control.

\section{INTRODUCTION}

Fundamental performance limitations in referencetracking for linear systems using feedback control have been quantified with classical Bode integrals and as the limits of cheap optimal control performance [Kwakernaak and Sivan, 1972; Middleton, 1991; Qiu and Davison, 1993; Seron et al., 1999; Chen et al., 2000]. In the absence of unstable zero dynamics (non-minimum phase zeros) and if the system is right invertible, perfect tracking of any reference signal is possible, that is, the $\mathcal{L}_{2}$ norm of the tracking error can be made arbitrarily small. However, this is not the case in the presence of unstable zero dynamics. A formula derived by Qiu and Davison [1993], see also [Su et al., 2003], shows that the tracking error increases as the signal frequencies approach those of the unstable zeros.

For step reference signals, Seron et al. [1999] reinterpreted the Qiu-Davison formula and general-

\footnotetext{
1 Supported by the National Science Foundation under Grant No. ECS-0228846.

2 Supported by the National Science Foundation under Grant No. ECS-0242798.
}

ized it to a class of nonlinear systems under the assumption that the relative degree is one and the zero dynamics are anti-stable. They showed that the best attainable value of the $\mathcal{L}_{2}$ norm of the tracking error $e_{T}(t)$, denoted by $J_{T}$, is equal to the lowest control effort, measured by the $\mathcal{L}_{2}$ norm, needed to stabilize the zero dynamics driven by $e_{T}(t)$. It is its role as a stabilizing control input that prevents the output $y(t)$ from perfect tracking. Extensions to non-right-invertible systems are given in [Woodyatt et al., 2002; Braslavsky et al., 2002].

In this paper we show that these results hold for the problem of tracking any reference signal generated by a known exosystem. The zero dynamics to be stabilized are those of the error system driven by $e_{T}(t)$. As before, the best attainable value of $J_{T}$ is the lowest energy needed to stabilize the zero-dynamics.

Path-following problems are concerned with the design of control laws that drive an object (robot arm, mobile robot, ship, aircraft, etc.) to reach and follow a geometric path. A secondary goal is to force the object moving along the path to satisfy some additional dynamic specification such 
as follow the path with some desired velocity. A common approach to the path-following problem is to parameterize the geometric path $y_{d}$ by a path variable $\theta$ and then select a timing law for $\theta$, [Hauser and Hindman, 1995; Al-Hiddabi and McClamroch, 2002; Skjetne et al., 2004; Aguiar et al., 2004; Aguiar et al., 2004a; Aguiar and Hespanha, 2004b]. A framework for path-following as a method to avoid some limitations in referencetracking was described in [Aguiar et al., 2004a]. The key idea is to use $\theta$ as an additional control input to stabilize the unstable zero-dynamics while the original control variables keep the system on the path.

We show that for a class of nonlinear systems the fundamental performance limitations imposed on reference-tracking by unstable zero dynamics do not apply to the path-following problem. Furthermore, the same is true for the speed-assigned path-following problem in which a speed assignment is required to be satisfied asymptotically or in finite time.

In Section 2 we formulate the reference-tracking and path-following problems. In Section 3 we briefly review our recent results for non-minimum phase linear systems, [Aguiar et al., 2004], showing that the path-following problems can be solved with arbitrarily small $\mathcal{L}_{2}$ norm of the pathfollowing error. Section 4 presents the main results of the paper. Concluding remarks are given in Section 5 .

\section{REFERENCE-TRACKING AND PATH-FOLLOWING PROBLEMS}

\subsection{Reference-tracking}

The classical reference-tracking problem is to design a feedback controller such that the closedloop system is asymptotically stable and, for any reference signal $r(t)$ in a prescribed family, the plant output converges to $r(t)$.

For linear systems

$$
\dot{x}=A x+B u, \quad y=C x+D u,
$$

$x \in \mathbb{R}^{n}, u \in \mathbb{R}^{m}, y \in \mathbb{R}^{q}$, and reference signals $r(t) \in \mathbb{R}^{q}$ generated by a known exosystem

$$
\dot{w}=S w, \quad r=Q w,
$$

Davison [1976] and Francis [1977] show that the so-called servomechanism or regulator problem is solvable if and only if $(A, B)$ is stabilizable, $(C, A)$ is detectable, the number of inputs is at least as large as the number of outputs $(m \geq q)$, and the zeros of $(A, B, C, D)$ do not coincide with the eigenvalues of $S$. The internal model approach, [Francis and Wonham, 1976; Francis, 1977], designs the reference-tracking controller

$$
u(t)=K x(t)+(\Gamma-K \Pi) w(t),
$$

where $K$ is such that $(A+B K)$ is Hurwitz, and $\Pi$ and $\Gamma$ satisfy

$$
\begin{aligned}
\Pi S & =A \Pi+B \Gamma, \\
0 & =C \Pi+D \Gamma-Q .
\end{aligned}
$$

Then, the tracking error defined as

$$
e_{T}(t):=y(t)-r(t)
$$

converges to zero, and the transients

$$
\tilde{x}:=x-\Pi w, \quad \tilde{u}:=u-\Gamma w
$$

are governed by $\dot{\tilde{x}}=(A+B K) \tilde{x}, \tilde{u}=K \tilde{x}$.

For the nonlinear regulator problem

$$
\begin{array}{llrl}
\dot{x} & =f(x, u), & & y=h(x, u), \\
\dot{w} & =s(w), & & r=q(w),
\end{array}
$$

where $f(0,0)=0, x \in \mathbb{R}^{n}$ is the state, $u \in \mathbb{R}^{m}$ the control, $y \in \mathbb{R}^{q}$ the output, $w \in \mathbb{R}^{p}$ the state of the exosystem, and $r \in \mathbb{R}^{q}$ the reference signal, Isidori and Byrnes [1990] show that it is solvable if and only if there exist smooth maps $\Pi(w)$, and $c(w)$, satisfying

$$
\begin{array}{ll}
\frac{\partial \Pi}{\partial w} s(w)=f(\Pi(w), c(w)), & \Pi(0)=0, \\
h(\Pi(w), c(w))-q(w)=0, & c(0)=0 .
\end{array}
$$

Krener [1992] presents necessary and sufficient conditions for local solvability of (6) when either the exosystem has a semisimple pole structure or the plant has a semisimple zero structure.

\subsection{Path-following}

In path-following, the output $y(t)$ is required to reach and follow a geometric path $y_{d}(\theta)$, where $\theta \in \mathbb{R}$ is the path variable. In this paper, the path $y_{d}(\theta)$ is assumed to be generated by the exosystem

$$
\begin{aligned}
\frac{d}{d \theta} w(\theta) & =s(w(\theta)), \quad w\left(\theta_{0}\right)=w_{0} \\
y_{d}(\theta) & =q(w(\theta)),
\end{aligned}
$$

where $w \in \mathbb{R}^{p}, y_{d} \in \mathbb{R}^{q}$, and $\theta_{0}:=\theta(0)$. For a given timing law $\theta(t)$, the path-following error is defined as

$$
e_{P}(t):=y(t)-y_{d}(\theta(t)) .
$$

We consider the following two path-following problems:

Geometric path-following: For a desired path $y_{d}(\theta)$, design a controller that achieves:

i) boundedness: the state $x(t)$ is uniformly bounded for all $t \geq 0$ and for every $(x(0), w(\theta(0)))=\left(x_{0}, w_{0}\right)$, in some neighborhood of $(0,0)$,

ii) error convergence: the path-following error $e_{P}(t)$ converges to zero as $t \rightarrow \infty$, and

iii) forward motion: $\dot{\theta}(t)>c$ for all $t \geq 0$, where $c$ is a positive constant.

Speed-assigned path-following: In addition to the geometric path-following task, a constant speed $v_{d}>0$ is assigned and it is required that either $\dot{\theta}(t) \rightarrow v_{d}$ as $t \rightarrow \infty$, or $\dot{\theta}(t)=v_{d}$ for all $t \geq T$ and some $T \geq 0$.

As illustrated by Skjetne et al. [2004], these pathfollowing problems provide natural settings for many engineering applications. From a theoretical standpoint our main interest is to determine 
whether the freedom to select a timing law $\theta(t)$ can be used to achieve an arbitrarily small $\mathcal{L}_{2}$ norm of the path-following error, that is, whether $\delta^{\star}>0$ in

$$
\int_{0}^{\infty}\left\|e_{P}(t)\right\|^{2} d t \leq \delta^{\star}
$$

can be made arbitrarily small.

\section{LIMITS OF PERFORMANCE FOR LINEAR SYSTEMS}

\subsection{Reference-tracking}

In reference-tracking, to satisfy the requirement that

$$
\int_{0}^{\infty}\left\|e_{T}(t)\right\|^{2} d t \leq \delta^{\star}
$$

for an arbitrary $\delta^{\star}>0$, while keeping the closedloop system stable, the zeros of (1) must lie in the open left half-plane $\mathbb{C}^{-},[$Kwakernaak and Sivan, 1972].

The non-minimum phase zeros, that is the zeros in $\mathbb{C}^{+}$, impose a fundamental limitation on the attainable tracking performance (10). This is revealed by the fact, [Kwakernaak and Sivan, 1972], that the limit $J_{T}$, as $\epsilon \rightarrow 0$, of the optimal value of the cheap control cost functional

$$
J_{\epsilon}:=\min _{\tilde{u}} \int_{0}^{\infty}\left[\left\|e_{T}(t)\right\|^{2}+\epsilon^{2}\|\tilde{u}(t)\|^{2}\right] d t
$$

with $\tilde{u}$ defined in (3), is strictly positive. Qiu and Davison [1993] showed that the locations of the zeros in $\mathbb{C}^{+}$determine the best attainable performance, that is the value of $J_{T}$.

Theorem 1. (Qiu and Davison [1993]). Let $r(t):=$ $\eta_{1} \sin \omega t+\eta_{2} \cos \omega t, x(0)=0$, and assume that (1) is right-invertible, has no zeros at $j \omega$, and its nonminimum phase zeros are $z_{1}, z_{2}, \ldots, z_{p}$. Then the best attainable performance $J_{T}:=\lim _{\epsilon \rightarrow 0} J_{\epsilon}$ is given by

$$
J_{T}=\eta^{\prime} M \eta, \quad \eta=\operatorname{col}\left(\eta_{1}, \eta_{2}\right),
$$

where $M$ is positive semi-definite and its trace satisfies

$$
\operatorname{trace} M=\sum_{i=1}^{p}\left(\frac{1}{z_{i}-j \omega}+\frac{1}{z_{i}+j \omega}\right) .
$$

For more general reference signals, [Su et al., 2003] give explicit formulas which show the dependence of $J_{T}$ on the non-minimum phase zeros and their frequency-dependent directional information.

\subsection{Path-following}

In contrast to reference-tracking, the attainable performance for path-following is not limited by non-minimum phase zeros, [Aguiar et al., 2004]. Let the desired path

$$
y_{d}(\theta):=\sum_{k=1}^{n_{d}}\left[a_{k} e^{j \omega_{k} \theta}+a_{k}^{\star} e^{-j \omega_{k} \theta}\right],
$$

where the $\omega_{k}>0$ are real numbers and the $a_{k}$ are non-zero complex vectors, be generated by exosystem (7). Considering, as in [Qiu and Davison, 1993; Woodyatt et al., 2002; Braslavsky et al., 2002; Su et al., 2003], that $x(0)=0$, [Aguiar et al., 2004] prove:

Theorem 2. (Aguiar et al. [2004]). Consider the geometric path-following problem for (1) where $(A, B)$ is stabilizable. Then for any given positive constant $\delta^{\star}$ there exist constant matrices $K$ and $L$, and a timing law $\theta(t)$ such that the feedback law

achieves (9).

$$
u(t)=K x(t)+L w(\theta(t))
$$

It is important to stress that the stabilizability of $(A, B)$ is the only condition (necessary and sufficient) for the solvability of the geometric path-following problem using (13).

Furthermore, an arbitrarily small $\mathcal{L}_{2}$ norm of the path-following error is attainable even when the speed assignment $v_{d}$ is specified beforehand.

Theorem 3. (Aguiar et al. [2004]). For the speedassigned path-following problem, let $v_{d}$ be specified so that the eigenvalues of $v_{d} S$ do not coincide with the zeros of $(4)$, and assume that $(A, B)$ is stabilizable. Then, (9) can be satisfied for any $\delta^{\star}>0$ with a timing law $\theta(t)$ and a controller of the form (13) but with time-varying piecewiseconstant matrices $K$ and $L$.

\section{LIMITS OF PERFORMANCE FOR NONLINEAR SYSTEMS}

In the first part of this section, we present an internal model analog of the results in [Seron et al., 1999; Braslavsky et al., 2002] for the referencetracking problem. In the second part, we present our main result for the path-following problem. We show that, in contrast to reference-tracking, the path-following problems can be solved with arbitrarily small $\mathcal{L}_{2}$ norm of the path-following error.

\subsection{Reference-tracking}

We consider the class of nonlinear systems which are locally diffeomorphic to systems in strictfeedback form ${ }^{3}$ :

$$
\begin{aligned}
\dot{z}= & f_{0}(z)+g_{0}(z) \xi_{1}, \\
\dot{\xi}_{1}= & f_{1}\left(z, \xi_{1}\right)+g_{1}\left(z, \xi_{1}\right) \xi_{2} \\
& \vdots \\
\dot{\xi}_{r_{d}} & =f_{r_{d}}\left(z, \xi_{1}, \ldots, \xi_{r_{d}}\right)+g_{r_{d}}\left(z, \xi_{1}, \ldots, \xi_{r_{d}}\right) u \\
y & =\xi_{1}
\end{aligned}
$$

where $z \in \mathbb{R}^{n_{z}}, \xi:=\operatorname{col}\left(\xi_{1}, \ldots, \xi_{r_{d}}\right), \xi_{i} \in \mathbb{R}^{m}$, $\forall i \in\left\{1, \ldots, r_{d}\right\}, u \in \mathbb{R}^{m}$, and $y \in \mathbb{R}^{m} \cdot f_{i}(\cdot)$

3 When convenient we use the compact form (4) for (14). In that case, $f(\cdot)$ denotes the vector field described by the right-hand-side of $(14 \mathrm{a})-(14 \mathrm{~b}), h(\cdot)$ the output map described by (14c), and $x=\operatorname{col}\left(z, \xi_{1}, \ldots, \xi_{r_{d}}\right)$. 
and $g_{i}(\cdot)$ are $\mathcal{C}^{k}$ functions of their arguments (for some large $k), f_{i}(0, \ldots, 0)=0$, and the matrices $g_{i}(\cdot), \forall i \in\left\{1, \ldots, r_{d}\right\}$ are always nonsingular. We assume that initially the system is at rest, $(z, \xi)=(0,0)$.

When the reference-tracking problem is solvable, there exist maps $\Pi=\operatorname{col}\left(\Pi_{0}, \ldots, \Pi_{r_{d}}\right), \Pi_{0}: \mathbb{R}^{p} \rightarrow$ $\mathbb{R}^{n_{z}}, \Pi_{i}: \mathbb{R}^{p} \rightarrow \mathbb{R}^{m}, \forall i \in\left\{1, \ldots, r_{d}\right\}$, and $c: \mathbb{R}^{p} \rightarrow \mathbb{R}^{m}$ that satisfy (6). The following locally diffeomorphic change of coordinates

$$
\begin{aligned}
\tilde{z} & =z-\Pi_{0}(w), \\
\tilde{\xi} & :=\operatorname{col}\left(\tilde{\xi}_{1}, \ldots, \tilde{\xi}_{r_{d}}\right), \\
\tilde{\xi}_{i} & =\xi_{i}-\Pi_{i}(w), \quad i=1, \ldots, r_{d} \\
\tilde{u} & =u-c(w),
\end{aligned}
$$

transforms the system (14) into the error system

$$
\begin{aligned}
\dot{\tilde{z}}= & \tilde{f}_{0}(\tilde{z}, w)+\tilde{g}_{0}(\tilde{z}, w) e_{T}, \\
\dot{\tilde{\xi}}_{1}= & \tilde{f}_{1}\left(\tilde{z}, \tilde{\xi}_{1}, w\right)+\tilde{g}_{1}\left(\tilde{z}, \tilde{\xi}_{1}, w\right) \tilde{\xi}_{2}, \\
& \vdots \\
\dot{\tilde{\xi}}_{r_{d}} & =\tilde{f}_{r_{d}}\left(\tilde{z}, \tilde{\xi}_{1}, \ldots, \tilde{\xi}_{r_{d}}, w\right)+\tilde{g}_{r_{d}}\left(\tilde{z}, \tilde{\xi}_{1}, \ldots, \tilde{\xi}_{r_{d}}, w\right) \tilde{u}, \\
e_{T} & =\tilde{\xi}_{1},
\end{aligned}
$$

where

$$
\begin{aligned}
\tilde{f}_{0}: & =f_{0}\left(\tilde{z}+\Pi_{0}(w)\right)-f_{0}\left(\Pi_{0}(w)\right) \\
& +\left[g_{0}\left(\tilde{z}+\Pi_{0}(w)\right)-g_{0}\left(\Pi_{0}(w)\right)\right] q(w), \\
\tilde{g}_{0}:= & g_{0}\left(\tilde{z}+\Pi_{0}(w)\right),
\end{aligned}
$$

$\tilde{f}_{0}(0, w)=0, \tilde{g}_{0}(\tilde{z}, 0)=g_{0}(\tilde{z})$, and $\tilde{f}_{i}(\cdot), \tilde{g}_{i}(\cdot), i=$ $1, \ldots, r_{d_{\tilde{f}}}$ are appropriately defined functions that satisfy $\tilde{f}_{i}(0, \ldots, 0, w)=0$ and $\tilde{g}_{i}\left(\tilde{z}, \ldots, \tilde{\xi}_{i}, 0\right)=$ $g_{i}\left(\tilde{z}, \ldots, \tilde{\xi}_{i}\right)$.

Our analysis makes use of the following two optimal control problems.

Cheap control problem: For the system consisting of the error system (19) and the exosystem (5) with initial condition $(\tilde{z}(0), \tilde{\xi}(0), w(0))=$ $\left(\tilde{z}_{0}, \tilde{\xi}_{0}, w_{0}\right)$, find the optimal feedback law $\tilde{u}=$ $\alpha_{\delta, \epsilon}^{c c}(\tilde{z}, \tilde{\xi}, w)$ that minimizes the cost functional

$$
\frac{1}{2} \int_{0}^{\infty}\left(\left\|e_{T}(t)\right\|^{2}+\delta\|\tilde{z}(t)\|^{2}+\epsilon^{2 r_{d}}\|\tilde{u}(t)\|^{2}\right) d t
$$

for $\delta>0, \epsilon>0$. We denote by $J_{\delta, \epsilon}^{c c}\left(\tilde{z}_{0}, \tilde{\xi}_{0}, w_{0}\right)$ the corresponding optimal value. The best-attainable cheap control performance for reference-tracking is then

$$
J_{T}:=\lim _{(\delta, \epsilon) \rightarrow 0} J_{\delta, \epsilon}^{c c}\left(\tilde{z}_{0}, \tilde{\xi}_{0}, w_{0}\right) .
$$

As shown in [Krener, 2001], in some neighborhood of $(0,0,0)$ and for every $\delta>0, \epsilon>0$, the value $J_{\delta, \epsilon}^{c c}(\cdot, \cdot, \cdot)$ is $\mathcal{C}^{k-2}$ under the following assumption:

Assumption 1. The linearization around $(z, \xi)=$ $(0,0)$ of system $(14)$ is stabilizable and detectable, and the linearization around $w=0$ of the exosystem (5) is stable.
Minimum-energy problem: For the system

$$
\begin{aligned}
\dot{\tilde{z}} & =\tilde{f}_{0}(\tilde{z}, w)+\tilde{g}_{0}(\tilde{z}, w) e_{T}, & \tilde{z}(0) & =z_{0}, \\
\dot{w} & =s(w), & w(0) & =w_{0},
\end{aligned}
$$

with $e_{T}$ viewed as the input, find the optimal feedback law $e_{T}=\alpha_{\delta}^{m e}(\tilde{z}, w)$ that minimizes the cost

$$
\frac{1}{2} \int_{0}^{\infty}\left(\delta\|\tilde{z}(t)\|^{2}+\left\|e_{T}(t)\right\|^{2}\right) d t
$$

for $\delta>0$. We denote by $J_{\delta}^{m e}\left(\tilde{z}_{0}, w_{0}\right)$ the corresponding optimal value. Under Assumption 1, $J_{\delta}^{m e}(\cdot, \cdot)$ is $\mathcal{C}^{k-2}$ in some neighborhood of $(0,0)$.

Our analysis reveals that $J_{T}$ is equal to the least control effort needed to stabilize the corresponding zero dynamics system (22) driven by the tracking error $e_{T}$.

Theorem 4. Suppose that Assumption 1 holds and that (6) has a solution in some neighborhood of $w=0$. Then, for any $(\tilde{z}(0), \tilde{\xi}(0), w(0))=$ $\left(\tilde{z}_{0}, \tilde{\xi}_{0}, w_{0}\right)$ in some neighborhood of $(0,0,0)$ there exists a solution to the cheap control problem and

$$
J_{T}=\lim _{\delta \rightarrow 0} J_{\delta}^{m e}
$$

Proof. Under Assumption 1 and from the formulations of the cheap control and minimum-energy problems, we conclude that for everry $\delta>0, \epsilon>0$, and every initial condition $\left(\tilde{z}_{0}, \tilde{\xi}_{0}, w_{0}\right)$ in some neighborhood of $(0,0,0)$, the values $J_{\delta}^{m e}\left(\tilde{z}_{0}, w_{0}\right)$ and $J_{\delta, \epsilon}^{c c}\left(\tilde{z}_{0}, \tilde{\xi}_{0}, w_{0}\right)$ exist and satisfy

$$
J_{\delta}^{m e}\left(\tilde{z}_{0}, w_{0}\right) \leq J_{\delta, \epsilon}^{c c}\left(\tilde{z}_{0}, \tilde{\xi}_{0}, w_{0}\right) .
$$

On the other hand, from Lemma 7 in Appendix we have

$$
J_{\delta, \epsilon}^{c c}\left(\tilde{z}_{0}, \tilde{\xi}_{0}, w_{0}\right) \leq J_{\delta}^{m e}\left(\tilde{z}_{0}, w_{0}\right)+O(\epsilon) .
$$

Therefore, from (25)-(26) we conclude that

$J_{\delta}^{m e}\left(\tilde{z}_{0}, w_{0}\right) \leq J_{\delta, \epsilon}^{c c}\left(\tilde{z}_{0}, \tilde{\xi}_{0}, w_{0}\right) \leq J_{\delta}^{m e}\left(\tilde{z}_{0}, w_{0}\right)+O(\epsilon)$

The result (24) follows from this and (21) as one makes $(\delta, \epsilon) \rightarrow 0$.

\subsection{Path-following}

For path-following, we define the correspondent cheap control problem by replacing $e_{T}$ with $e_{P}$ in (20). We then show that, in contrast to reference-tracking, the path-following problem can be solved with arbitrarily small $\mathcal{L}_{2}$ norm of $e_{P}$.

We let the vector field $s(w)$ and the output map $q(w)$ of the exosystem (7) be linear, $s(w)=S w$, $q(w)=Q w$, such that all eigenvalues of $S \in \mathbb{R}^{p \times p}$ are non-zero and semisimple.

Theorem 5. Assume that (6) has a solution when $s(w)=v_{d} S w$, for $v_{d}$ almost everywhere on $(0, \infty)$. Then, for every $w(\theta(0))=w_{0}$ in a neighborhood around $w=0$, there exist a timing law for $\theta(t)$ and a feedback law

$$
u=c(w)+\alpha_{\delta, \epsilon}(z, \xi, w)
$$

which solve the geometric path-following and satisfy (9) for every $\delta^{\star}>0$. 
Proof. With the timing law

$$
\dot{\theta}(t)=v_{d}, \quad \theta(0)=0,
$$

and $v_{d}>0$ a constant to be selected later, the path-following problem becomes the tracking problem of $r(t)$ generated by

$$
\dot{w}(t)=v_{d} S w(t), \quad r(t)=Q w(t),
$$

which, upon the substitution in (6), yields

$$
\begin{gathered}
\frac{\partial \Pi}{\partial w} v_{d} S w=f(\Pi(w), c(w)), \\
h(\Pi(w), c(w))-Q w=0 .
\end{gathered}
$$

The function $c(w)$, used in the feedback law (27), solves (30), while $\alpha_{\delta, \epsilon}(z, \xi, w)$ minimizes (20) for the error system (19) together with the exosystem (29) and some small $\delta>0, \epsilon>0$. With the timing law (28), Theorem 4 allows us to conclude that as $(\epsilon, \delta) \rightarrow 0$ we have $J_{P}=\lim _{\delta \rightarrow 0} J_{\delta}^{m e}$.

To prove that $J_{\delta}^{m e}$ can be made arbitrarily small by selecting a sufficiently large $v_{d}$, we use Lemma 8 in Appendix. It shows that for the minimumenergy problem and every initial condition in some neighborhood of $(\tilde{z}, w)=(0,0)$, there exist a sufficiently small $\delta>0$ in (23) and a feedback law $e_{T}=\hat{\alpha}_{\delta}^{m e}(\tilde{z}, w)$ for which $J_{\delta}^{m e}\left(\tilde{z}_{0}, w_{0}\right)$ is bounded by

$$
J_{\delta}^{m e}\left(\tilde{z}_{0}, w_{0}\right) \leq \frac{1}{2} \tilde{z}_{0}^{\prime} P_{0} \tilde{z}_{0},
$$

where $P_{0}>0$ does not depend on $v_{d}$. Observing that $\tilde{z}_{0}=\Pi_{0}\left(w_{0}\right)$, since $z(0)=0$, the proof is completed using Lemma 9 in Appendix which establishes that $\left\|\Pi_{0}\left(w_{0}\right)\right\|$ can be made arbitrarily small by choosing a sufficiently large $v_{d}$.

Next we show that an arbitrarily small $\mathcal{L}_{2}$ norm of the path-following error is attainable even when the speed $v_{d}$ is specified beforehand.

Theorem 6. Consider the speed-assigned pathfollowing problem with $v_{d}$ specified so that (30) has a solution in some neighborhood of $w=0$. Then, (9) can be satisfied for any $\delta^{\star}>0$ with a suitable timing law $\theta(t)$ and a controller of the form (27) with time-varying piecewise-continuous maps $c(w)$ and $\alpha(z, \xi, w)$.

Proof. To construct a path-following controller that satisfies (9) we start with

$$
\begin{aligned}
& u=c_{\sigma}(w)+\alpha_{\sigma}(z, \xi, w), \\
& \dot{\theta}=v_{\sigma},
\end{aligned}
$$

where for each positive constant $v_{\ell}, \ell \in \mathcal{I}:=$ $\{0,1,2, \ldots N\}$, the maps $\Pi_{\ell}:=\operatorname{col}\left(\Pi_{\ell_{0}}, \Pi_{\ell_{\xi}}\right)$, $\Pi_{\ell_{\xi}}:=\operatorname{col}\left(\Pi_{\ell_{\xi_{1}}}, \ldots, \Pi_{\ell_{\xi_{r_{d}}}}\right), \Pi_{\ell_{0}}: \mathbb{R}^{p} \rightarrow R^{n_{z}}$, $\Pi_{\ell_{i}}: \mathbb{R}^{p} \rightarrow R^{m}, i=1, \ldots, r_{d}$, and $c_{\ell}: \mathbb{R}^{p} \rightarrow \mathbb{R}^{m}$ satisfy

$$
\begin{gathered}
\frac{\partial \Pi_{\ell}}{\partial w} v_{\ell} S w=f\left(\Pi_{\ell}(w), c_{\ell}(w)\right), \\
h\left(\Pi_{\ell}(w), c_{\ell}(w)\right)-Q w=0,
\end{gathered}
$$

and $\sigma(t):\left[t_{0}:=0, \infty\right) \rightarrow \mathcal{I}$, is the piecewise constant switching signal

$$
\sigma(t)=\left\{\begin{array}{l}
i, \quad t_{i} \leq t<t_{i+1}, i=0, \ldots, N-1 \\
N, t \geq t_{N}
\end{array}\right.
$$

Each $\alpha_{\ell}(z, \xi, w)$ is the optimal feedback-law that minimizes

$\int_{0}^{\infty}\left(\left\|e_{P}\right\|^{2}+\delta\left\|z-\Pi_{\ell_{0}}(w)\right\|^{2}+\epsilon^{2 r_{d}}\left\|u-c_{\ell}(w)\right\|^{2}\right) d t$,

for some small $\delta>0, \epsilon>0$. Note that (31) is a speed-assignment path-following controller for which $\dot{\theta}(t)$ converges to $v_{N}=v_{d}$ in finite time.

We now prove that for any $\delta^{\star}>0,(9)$ can be satisfied by appropriate selection of a finite sequence $t_{0}, t_{1}, \ldots, t_{N}$ together with $\left(v_{0}, \Pi_{0}, \alpha_{0}, c_{0}\right),\left(v_{1}, \Pi_{1}\right.$, $\left.\alpha_{1}, c_{1}\right), \ldots,\left(v_{N}, \Pi_{N}, \alpha_{N}, c_{N}\right)$ used in the feedback controller (31). To this end, we show in Lemma 10 in Appendix that $J_{P}$ is bounded by

$$
\begin{aligned}
J_{P} \leq & \frac{1}{2} \tilde{z}_{0}^{\prime} P_{0} \tilde{z}_{0}+\gamma \frac{\lambda_{\max }\left(P_{0}\right)}{2} \sum_{\ell=1}^{N}\left(v_{\ell-1}-v_{\ell}\right)^{2} \\
& +\lambda_{\max }\left(P_{0}\right) \sum_{\ell=1}^{N} \tilde{z}_{\ell-1}\left(t_{\ell}\right)^{\prime}\left[\bar{z}_{\ell-1}\left(t_{\ell}\right)-\bar{z}_{\ell}\left(t_{\ell}\right)\right] \\
& +\frac{\lambda_{\max }\left(P_{0}\right)}{2} \sum_{\ell=1}^{N}\left\|\tilde{z}_{\ell-1}\left(t_{\ell}\right)\right\|^{2}
\end{aligned}
$$

where $\lambda_{\max }\left(P_{0}\right)$ denotes the maximum eigenvalue of $P_{0}>0, \gamma$ is a positive constant, $\tilde{z}_{0}:=\tilde{z}(0)$, $\bar{z}_{\ell}:=\Pi_{\ell_{0}}(w)$, and the transient $\tilde{z}_{\ell}:=z-\Pi_{\ell_{0}}(w)$ converges to zero as $t \rightarrow \infty$.

We show that each term of (33) is upper-bounded by $\frac{\delta^{\star}}{4}$ so that $J_{P} \leq \delta^{\star}$. Applying the same arguments as in Theorem 5, the first term in (33) is bounded by $\frac{\delta^{\star}}{4}$ using a sufficiently large $v_{0}$. To prove that the second term in (33) is smaller than $\frac{\delta^{\star}}{4}$, we select the parameters $v_{\ell}, \ell \in \mathcal{I}$ to satisfy

$$
v_{\ell-1}-v_{\ell}=\mu, \quad v_{N}=v_{d}, \quad \ell=1,2, \ldots, N
$$

where $\mu:=\frac{2 \delta^{\star}}{\gamma \lambda_{\max }\left(P_{0}\right)\left(v_{0}-v_{N}\right)}$, and $N:=\frac{v_{0}-v_{N}}{\mu}$. Then

$$
\begin{array}{r}
\gamma \frac{\lambda_{\max }\left(P_{0}\right)}{2} \sum_{\ell=1}^{N}\left(v_{\ell-1}-v_{\ell}\right)^{2} \leq \gamma \frac{\lambda_{\max }\left(P_{0}\right)}{2} N \mu^{2} \\
=\gamma \frac{\lambda_{\max }\left(P_{0}\right)}{2}\left(v_{0}-v_{N}\right) \mu=\frac{\delta^{\star}}{4} .
\end{array}
$$

The above selection for the $v_{\ell}, \ell \in \mathcal{I}$, it is made under the constraint that the reference-tracking problem for the signal $r(t)$ generated by (29) with $v_{d}$ replaced by $v_{\ell}$ is solvable. This can always be satisfied by appropriately adjusting $v_{0}$. Finally, for any given $N$, each of the last two terms in (33) can be made smaller than $\frac{\delta}{4}$ by choosing $t_{\ell}$, $\ell=1,2, \ldots, N$ sufficiently large.

\section{CONCLUSIONS}

This paper demonstrates that the task of following a geometric path $y_{d}(\theta)$ is less restrictive than the task of tracking a reference signal $r(t)$. The reference-tracking problem is subjected to the limitations imposed by the unstable zerodynamics, a nonlinear analog of the Bode's limitations caused by non-minimum phase zeros. Our analysis revealed that the limitation is due to 
the need to stabilize the zero-dynamics by the tracking error, which therefore prevents the output $y(t)$ from achieving perfect tracking. In pathfollowing one has available an additional degree of freedom to select a timing law $\theta(t)$ with which a prescribed path $y_{d}(\theta)$ will be followed. In Theorems 5 and 6 , we prove that with an appropriate choice of $\theta(t)$ the $\mathcal{L}_{2}$ norm of the path-following error can be made arbitrarily small, that is, the path-following problem is not subjected to the limitations of reference-tracking. This conceptual result may be of practical significance, because the path-following formulation is convenient for many applications. Design of path-following controllers for non-minimum phase systems is a topic of current research, [Dačić et al., 2004].

\section{Appendix A}

Due to space limitations, the proofs of the following lemmas are omitted. These can be found in [Aguiar et al., 2004c].

Lemma 7. Suppose that Assumption 1 holds. For every initial condition $\left(\tilde{z}_{0}, \tilde{\xi}_{0}, w_{0}\right)$ for the error system (19) and the exosystem (5) in some neighborhood of $(0,0,0)$, and every $\delta>0$, there exist a sufficiently small $\epsilon>0$ and feedback law $\tilde{u}=\hat{\alpha}_{\delta, \epsilon}^{c c}(\tilde{z}, \tilde{\xi}, w)$ for which the value of (20) does not exceed

$$
J_{\delta}^{m e}\left(\tilde{z}_{0}, w_{0}\right)+O(\epsilon)
$$

Lemma 8. Consider the minimum-energy problem formulated in Section 4. For every initial condition $(\tilde{z}(0), w(0))=\left(\tilde{z}_{0}, w_{0}\right)$ for $(22)$ in some neighborhood of $(0,0)$, there exist $\delta>0$ in $(23)$ and a feedback law $e_{T}=\hat{\alpha}_{\delta}^{m e}(\tilde{z}, w)$ for which (23) does not exceed

$$
\frac{1}{2} \tilde{z}_{0}^{\prime} P_{0} \tilde{z}_{0}
$$

where $P_{0}>0$ does not depend on $v_{d}$.

Lemma 9. In the reference-tracking problem for the nonlinear system (14) let the vector field $s(w)$ and the output map $q(w)$ of the exosystem (5) be $s(w)=v_{d} S w, q(w)=Q w$. Suppose that the eigenvalues of $S \in \mathbb{R}^{p \times p}$ are non-zero and semisimple, and that for some $v_{d}>0,(6)$ has a solution in some neighborhood of $w=0$. Then, for any $\rho>0$, there exists $v_{d}^{\star}>0$ such that the map $\Pi_{0}: \mathbb{R}^{p} \rightarrow \mathbb{R}^{n_{z}}$ satisfying

$$
\frac{\partial \Pi_{0}(w)}{\partial w} S w=\mu\left[f_{0}\left(\Pi_{0}(w)\right)+g_{0}\left(\Pi_{0}(w)\right) Q w\right],
$$

$\mu:=\frac{1}{v_{d}^{\star}}$, is bounded by

$$
\left\|\Pi_{0}(w)\right\| \leq \rho .
$$

Lemma 10. Under the conditions of Theorem 6 , the path-following controller (31) ensures that there exists $\gamma>0$ such that $J_{P}$ satisfies (33).

\section{REFERENCES}

Aguiar, A. P., J. P. Hespanha and P. Kokotović (2004). Path-following for non-minimum phase systems removes performance limitations. IEEE Trans. on Automat. Contr. 50(2), 234-239.
Aguiar, A. P., D. B. Dačić, J. P. Hespanha and P. Kokotović (2004a). Path-following or reference-tracking? An answer relaxing the limits to performance. In: Proc. of IAV2004 - 5th IFAC/EURON Symp. on Intel. Auton. Vehicles. Lisbon, Portugal.

Aguiar, A. P. and J. P. Hespanha (2004b). Logic-based switching control for trajectory-tracking and pathfollowing of underactuated autonomous vehicles with parametric modeling uncertainty. In: Proc. of the 2004 Amer. Contr. Conf.. Boston, MA, USA.

Aguiar, A. P., J. P. Hespanha and P. Kokotović (2004b). Limits of performance in reference-tracking and pathfollowing for nonlinear systems. Technical report. University of California, Santa Barbara.

Al-Hiddabi, S. A. and N. H. McClamroch (2002). Tracking and maneuver regulation control for nonlinear nonminimum phase systems: application to flight control. IEEE Trans. on Contr. Syst. Tech. 10(6), 780-792.

Braslavsky, J. H., R. H. Middleton and J. S. Freudenberg (2002). Cheap control performance of a class of nonright-invertible nonlinear systems. IEEE Trans. on Automat. Contr. 47(8), 1314-1319.

Chen, Jie, Li Qiu and O. Toker (2000). Limitations on maximal tracking accuracy. IEEE Trans. on Automat. Contr. 45(2), 326-331.

Dačić, D.B., M.V. Subbotin and P.V. Kokotović (2004). Path-following for a class of nonlinear systems with unstable zero dynamics. In: Proc. of the 43rd Conf. on Decision and Contr.. Paradise Island, Bahamas.

Davison, Edward J. (1976). The robust control of a servomechanism problem for linear time-invariant multivariable systems. IEEE Trans. on Automat. Contr. 21(1), 25-34.

Francis, B.A. (1977). The linear multivariable regulator problem. SIAM J. Contr. Optimization 15(3), 486505.

Francis, B.A. and W.M. Wonham (1976). The internal model principle of control theory. Automatica 12(5), 457-465.

Hauser, J. and R. Hindman (1995). Maneuver regulation from trajectory tracking: Feedback linearizable systems. In: Proc. IFAC Symp. Nonlinear Control Systems Design. Lake Tahoe, CA, USA. pp. 595-600.

Isidori, Alberto and Christopher I. Byrnes (1990). Output regulation of nonlinear systems. IEEE Trans. on Automat. Contr. 35(2), 131-140.

Krener, A.J. (1992). The construction of optimal linear and nonlinear regulators. pp. 301-322. Systems, Models and Feedback: Theory and Applications. A. Isidori and T. J. Tarn. Birkhauser, Boston.

Krener, A.J. (2001). The local solvability of a Hamilton Jacobi - Bellman PDE around a nonhyperbolic critical point. SIAM J. Contr. Optimization 39(5), 14611484.

Kwakernaak, H. and R. Sivan (1972). The maximal achievable accuracy of linear optimal regulators and linear optimal filters. IEEE Trans. on Automat. Contr. 17(1), 79-86.

Middleton, R. H. (1991). Trade-offs in linear control systems design. Automatica 27(2), 281-292.

Qiu, L. and E. J. Davison (1993). Performance limitations of nonminimum phase systems in the servomechanism problem. Automatica 29, 337-349.

Seron, M. M., J. H. Braslavsky, Petar V. Kokotović and David Q. Mayne (1999). Feedback limitations in nonlinear systems: From bode integrals to cheap control. IEEE Trans. on Automat. Contr. 44(4), 829-833.

Skjetne, R., T. I. Fossen and P. Kokotović (2004). Robust output maneuvering for a class of nonlinear systems. Automatica 40(3), 373-383.

$\mathrm{Su}$, Weizhou, Li Qiu and Jie Chen (2003). Fundamental performance limitations in tracking sinusoidal signals. IEEE Trans. on Automat. Contr. 48(8), 1371-1380.

Woodyatt, A.R., M.M. Seron, J.S. Freudenberg and R.H. Middleton (2002). Cheap control tracking performance for non-right-invertible systems. Int. J. of Robust and Nonlinear Control 12(15), 1253-1273. 